\title{
Magnetotransport and magnetic domain structure in compressively strained colossal magnetoresistance films
}

\author{
Yan Wu and Y. Suzuki ${ }^{\mathrm{a})}$ \\ Department of Materials Science and Engineering, Cornell University, Ithaca, New York 14853 \\ U. Rüdiger, J. Yu, and A. D. Kent \\ Department of Physics, New York University, New York, New York 10003
}

T. K. Nath and C. B. Eom

Department of Mechanical Engineering and Materials Science, Duke University, Durham, North Carolina 27708

(Received 18 May 1999; accepted for publication 12 August 1999)

\begin{abstract}
We have studied the magnetoresistance (MR) of compressively strained $\mathrm{La}_{0.7} \mathrm{Sr}_{0.3} \mathrm{MnO}_{3}$ (LSMO) films in various magnetic states in order to understand the role of magnetic domain structure on magnetotransport. In thin films of LSMO on (100) $\mathrm{LaAlO}_{3}$, the perpendicular magnetic anisotropy results in perpendicularly magnetized domains with fine scale $\sim 200 \mathrm{~nm}$ domain subdivision, which we image directly at room temperature using magnetic force microscopy. The main MR effects can be understood in terms of bulk colossal MR and anisotropic MR. We also find evidence for a small domain wall contribution to the MR, which is an order of magnitude larger than expected from a double exchange model.
\end{abstract}

The doped perovskite manganites have received an enormous amount of attention recently because they exhibit colossal magnetoresistance (CMR) and may be half metallic, with complete spin polarization at the Fermi level. For these reasons, they may find important uses in magnetoresistive devices, such as magnetic random access memory and sensors. As has been found in magnetoresistive devices based on transition metal ferromagnets, controlling the electronic transport and magnetic properties of these materials in thin film form will be essential to applications. Experimentally, the magnetic and transport properties of colossal magnetoresistance materials have been shown to be highly sensitive to microstructure as well as lattice distortions both in thin film and bulk form. Many groups have shown that properties such as Curie temperature, resistivity and magnetoresistance effect are extremely sensitive to chemical and hydrostatic pressure as well as lattice mismatch with an underlying substrate. ${ }^{1-11}$ Studies of bulk polycrystalline pellets, thin films of varying polycrystallinity and isolated grain boundaries have shown that the magnetoresistance is profoundly affected by transport across grain boundaries. ${ }^{2,12,13}$

Magnetic domain structure may also to lead to distinctive magnetotransport effects in thin films. Mathur et al. report that the measured resistivity of a magnetic domain wall is four orders of magnitude larger than that predicted by a simple double exchange picture. ${ }^{14}$ Wang et al. have also suggested that large low field magnetoresistance (MR), in ultrathin compressively strained doped manganite thin films, may be due to domain wall scattering. ${ }^{9}$ In order to address these questions in LSMO, we have prepared in-plane compressively strained films of LSMO on (001) LAO $(a=b=c$ $=3.79 \AA$ ) substrates using pulsed laser deposition. ${ }^{9}$ These films naturally split into stripe domains with length scales set

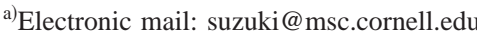

by the strain and film thickness. This enables us to study the effect of magnetic microstructure on MR in a systematic manner. In this letter, we have investigated the magnetics and magnetotransport of epitaxial $\mathrm{La}_{0.7} \mathrm{Sr}_{0.3} \mathrm{MnO}_{3}$ (LSMO) films. The main MR effects can be explained by CMR and anisotropic MR. We also find evidence for a small domain wall (DW) contribution to the MR, which is an order of magnitude larger than expected based purely on a double exchange picture. The results are consistent in magnitude with those reported by Mathur in constricted tracks of $\mathrm{La}_{0.7} \mathrm{Ca}_{0.3} \mathrm{MnO}_{3}$ films. ${ }^{14}$

The biaxial compressive strain of LSMO on LAO is predicted to give rise to a perpendicular anisotropy. ${ }^{5}$ In order to get a direct measure of the compressive strain imposed on the LSMO ( $a_{\text {bulk }}=b_{\text {bulk }}=c_{\text {bulk }}=3.88 \AA$ ) by the LAO substrate, we have measured the out-of-plane and in-plane lattice parameters of these films by normal and grazing incidence x-ray diffraction (GID) respectively. GID measurements probe the crystal structure to a depth of about $100 \AA$ below the surface. As the films are grown thicker, the in-plane lattice parameters relax toward the bulk value of $3.88 \AA$ A. Table I shows that very thin films have in-plane lattice parameters closer to the substrate lattice parameter than thicker LSMO and that complete relaxation of the film does not occur even at length scales of $1000 \AA$. . $^{15,16}$

Magnetic force microscopy (MFM) measurements re-

TABLE I. Grazing incidence diffraction (GID) measurements provide us with in-place lattice parameters that are indicative of the degree of compressive strains in films of various thicknesses.

\begin{tabular}{ccc}
\hline \hline Film thickness $(\AA)$ & $a_{\text {out-of-plane }}(\AA)$ & $a_{\text {in-plane }}(\AA)$ \\
\hline 280 & 3.947 & $3.832 \pm 0.005$ \\
600 & 3.939 & $3.841 \pm 0.003$ \\
1500 & 3.910 & $3.860 \pm 0.005$ \\
4500 & 3.897 & $3.880 \pm 0.005$ \\
\hline
\end{tabular}




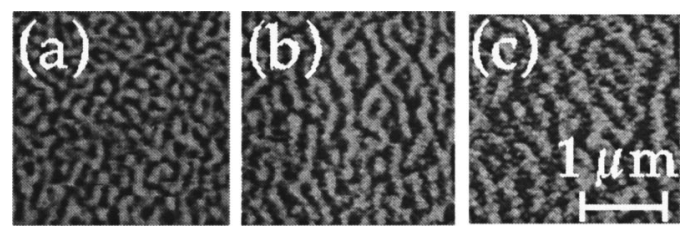

FIG. 1. Magnetic force microscopy in zero applied field of films of thickness: (a) $800 \AA$, (b) $1450 \AA$, and (c) $3750 \AA$ after a magnetic field had been applied in-plane (vertically in image). The domains are aligned in stripes along the direction of the demagnetization field. Domain size is seen to increase with increasing film thickness.

veal small scale "stripe" domains consistent with a predominantly perpendicular anisotropy. Figure 1 shows images taken in zero field at room temperature after the films have been demagnetized with an in-plane magnetic field. Stripe domains form due to a competition between the exchange, magnetostatic and magnetic anisotropy energies. The magnetic anisotropy must be sufficient to overcome the magnetostatic energy of the stripe domain state, which is significantly less than the maximum magnetostatic energy of $2 \pi M^{2}$ (where $M=$ magnetization) for a perpendicular magnetized single domain film. In the weak stripe domain limit, $Q=K / 2 M^{2}<1$ (where $K$ is the magnetic anisotropy), relevant to our films $(Q \sim 0.2)$, this condition amounts to the film thickness being greater than approximately the domain wall width $\left(=(A / K)^{1 / 2} \sim 300 \AA\right.$, where $A$ is the exchange stiffness). ${ }^{17} \mathrm{We}$ observe a trend toward increasing domain size with film thickness (Fig. 2), which reflects the basic scaling expected for the magnetic interactions with film thickness as well as a reduction in the magnetic anisotropy with film thickness, due to reduced average strain in thicker films. ${ }^{18}$ Since MFM images the magnetic structure of the surface, the domain structure is representative of domain structure averaged over approximately an exchange length $(\sim 500 \AA)$ below the surface, which is a significant fraction of the film thickness.

We have measured the hysteresis loops of (100) LSMO films on (100) LAO substrates. Magnetization loops with fields applied parallel and perpendicular to the film plane show zero remanence and are consistent with that of a weak stripe domain material. We can attribute the linear slope of the perpendicular magnetization curve (Fig. 2) to reversible domain wall motion, with saturation at a field of approximately half the film demagnetization field $\left(4 \pi M_{s}\right.$ $=4500 \mathrm{Oe}) .{ }^{19}$ The fact that the film saturates at a field less than the demagnetization field is due to energy gain associ-

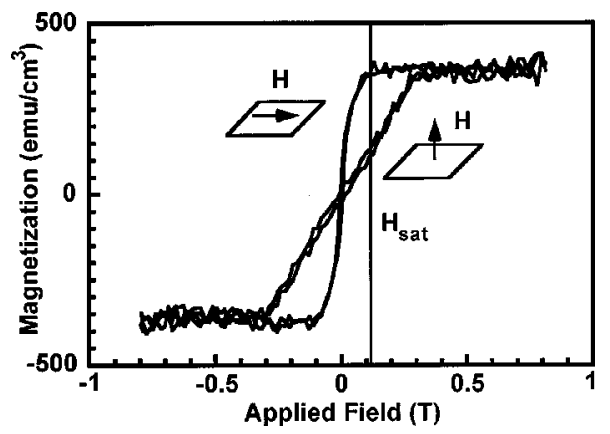

FIG. 2. Magnetization hysteresis loops of a $800 \AA$ LSMO/LAO film with field applied perpendicular and parallel to the plane of the film respectively.

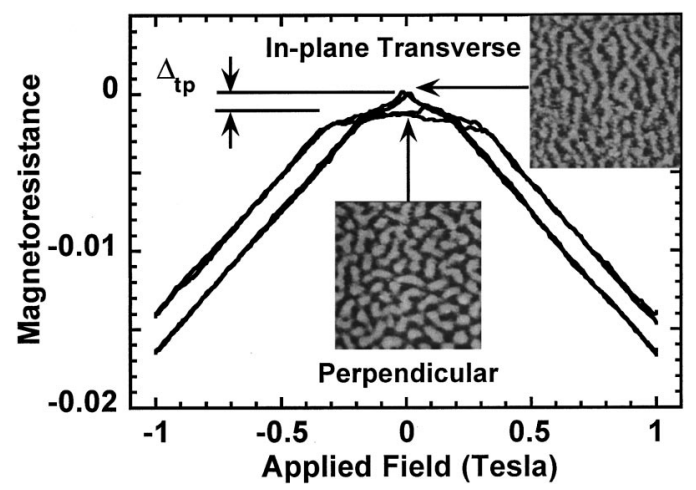

FIG. 3. Magnetoresistance vs applied field of a $800 \AA ̊$ LSMO/LAO at room temperature with the applied field perpendicular to the film plane as well as in the film plane and transverse to the current direction. The small difference in resistivity at $H=0$, labeled $\Delta_{t p}$, is the effect of domain configurations on resistivity. Insets indicate domain structure at zero field after saturation in the two directions.

ated with the erasure of domain walls. ${ }^{20}$ The in-plane hysteresis loop saturates at 1200 Oe and corresponds to domain rotation away from the easy axis. From this saturation field a magnetic anisotropy energy density of $2 \times 10^{5} \mathrm{ergs} / \mathrm{cm}^{3}$ is estimated for this film.

As the films become thicker, magnetization loops indicate an increasingly larger contribution from magnetic material that is less strained and hence have a smaller saturation field and magnetic anisotropy. Temperature-dependent measurements of the magnetization loops also confirm that the saturation fields and magnetic anisotropy increase with decreasing temperature as expected. These trends can be directly correlated to features in the magnetotransport.

We have also performed magnetotransport measurements on these compressively strained LSMO films. We can understand the salient features of the magnetotransport in terms of the magnetics. Figure 3 shows the magnetotransport measurements for an $800 \AA$ film. Let us first consider the configuration in which the field is applied perpendicular to the plane of the film. We observe a negative MR with a high field linear dependence on the applied field. We attribute this negative MR to the suppression of spin fluctuations at high fields (CMR). At lower fields, there is a sublinear deviation of the MR from the linear high field behavior, with the resistivity approximately field independent. The field at which the deviation occurs corresponds to the magnetic saturation field, $H_{\text {sat }}$ (see Fig. 2). As the field is lowered below $H_{\text {sat }}$, reversed magnetic domains form and, while the applied field is varying, the internal magnetic field $B$ is approximately constant and the CMR is suppressed.

The negative linear high field MR response for applied fields in-plane and transverse to the current is again associated with CMR. Here, the MR behavior for fields less than the saturation field is due to the rotation of the magnetization away from the in-plane applied field direction toward the film normal. Due to demagnetization effects the internal field is reduced as the moments rotate, which by CMR alone would lead to a superlinear deviation of the MR. However, the resistivity anisotropy in our films is such that the resistivity is smallest when the magnetization is perpendicular to the film plane and for this reason the MR deviation is sublinear. $^{21-23}$ 
The difference between the in-plane transverse and perpendicular MR at $H=0$ reflects the effect of domain configurations on film resistivity. This difference, $\Delta_{t p}$, is indicated in Fig. 3. After demagnetization with a perpendicular applied field, MFM images show that domains form in a "maze" configuration, so that DW structures are oriented randomly with respect to the current flow. In contrast, when films are demagnetized with an in-plane applied field, domains are elongated along the applied field direction (Fig. 1). Thus, for applied fields in-plane and transverse to the current, the DW structures at $H=0$ are arranged mainly perpendicular to the current. If domain wall scattering were significant, $\Delta_{t p}$ would be large since domain wall scattering would lead to significantly larger resistivities for current perpendicular to the DW structures (i.e., after in-plane transverse saturation). We are assuming that in the "maze" configuration a portion of the current is shunted by the domains and need not cross domain walls producing a smaller resistivity. We find that the difference, $\Delta_{t p}$, is on the order of $0.1 \%$ (Fig. 3). Differences of this order were also observed for other thickness films studied. The DW MR is then given by $\mathrm{MR}_{\text {interface }}=(d / \delta) \Delta_{p t}=3 \times 10^{-3}$ (or a specific resistivity of $\left.\rho_{\text {interface }}=(d / \delta) \Delta_{p t} \rho \delta=\Delta_{t p} \rho d \sim 1 \times 10^{-11} \Omega \mathrm{cm}^{2}\right)$, where $d$ is the domain size, $\delta$ is the domain wall width, and $\rho$ is the film resistivity. Within the double exchange picture, we can estimate a DW MR. Experimentally, the anisotropy is approximately $2 \times 10^{5} \mathrm{erg} / \mathrm{cm}^{3}$ and we can estimate the exchange interaction $J_{\mathrm{ex}} \sim 2.0 \times 10^{-15} \mathrm{erg}^{24}$ so that the domain wall width is about $320 \AA$ or 82 unit cells. Therefore within a double exchange picture, the adjacent Mn moments are misaligned by about $\theta_{12}=2^{\circ}$, thus resulting in a bandwidth reduced by $\cos \theta_{12} / 2$. If we assume that the carrier mean free path is on the order of the domain wall width, then we can the estimate the MR due to the domain wall to be 1.5 $\times 10^{-4}$. This value is more than order of magnitude smaller than the observed MR. ${ }^{25}$ A more detailed model of the domain wall MR, in double exchange ferromagnets, predicts a $1 \%-2 \%$ effect which is consistent with our measured MR. ${ }^{26}$ Finally, it is also interesting to note that a macroscopic mechanism based on the Hall effect (which produces current deflection near DWs) could also potentially lead to $\Delta_{t p}$ values of the order of those measured. ${ }^{27}$

The authors acknowledge Jonathan Sun for useful discussions. This work was supported in part by an NSF CAREER Award (Y.S.), a David and Lucile Packard Foundation Fellowship (Y.S., C.B.E.), NSF-MRSEC funds (Y.S.), DARPA-ONR Grant No. N00014-96-1-1207 (A.D.K.), and NSF-DMR 9802444 (C.B.E.). Some structural characteriza- tion was carried out at the central facilities of the Cornell Center for Materials Research (NSF-MRSEC).

${ }^{1}$ S. Jin, T. H. Tiefel, M. McCormack, H. M. O'Bryan, L. H. Chen, R. Ramesh, and D. Schurig, Appl. Phys. Lett. 67, 557 (1995).

${ }^{2}$ H. Y. Hwang, T. T. M. Palstra., S.-W. Cheong, and B. Batlogg, Phys. Rev. B 52, 15046 (1995).

${ }^{3}$ M. R. Ibarra, P. A. Algarabel, C. Marquina, J. Basco, and J. Garcia, Phys. Rev. Lett. 75, 3541 (1995).

${ }^{4}$ J. J. Neumeier, M. F. Hundley, J. D. Thompson, and R. H. Heffner, Phys. Rev. B 52, R7006 (1995).

${ }^{5}$ J. O'Donnell, M. S. Rzchowski, J. N. Eckstein, and I. Bosovic, Appl. Phys. Lett. 69, 1312 (1996); 72, 1775 (1998).

${ }^{6}$ Y. Suzuki, H. Y. Hwang, S.-W. Cheong, and R. B. van Dover, Appl. Phys. Lett. 71, 140 (1997).

${ }^{7}$ C. Kwon, K.-C. Kim, M. C. Robson, S. E. Lofland, S. M. Bhagat, T. Venkatesan, R. Ramesh, and R. D. Gomez, J. Magn. Magn. Mater. 172, 229 (1997).

${ }^{8}$ N.-C. Yeh, R. P. Vasquez, D. A. Beam, C.-C. Fu, J. Huynh, and G. Beach, J. Phys.: Condens. Matter 9, 3713 (1997).

${ }^{9}$ H. S. Wang and Qi Li, Appl. Phys. Lett. 73, 2360 (1998).

${ }^{10}$ T. K. Nath, R. A. Rao, D. Lavric, C. B. Eom, L. Wu, and F. Tsui, Appl. Phys. Lett. 74, 1615 (1999).

${ }^{11}$ K. A. Thomas, P. S. I. P. N. De Silva, L. F. Cohen, A. Hossain, M. Rajeswari, T. Venkatesan, R. Hiskes, and J. L. MacManus-Driscoll, J. Appl. Phys. 84, 3939 (1998).

${ }^{12}$ A. Gupta, G. Q. Gong, G. Xiao, P. R. Duncombe, P. Lecoeur, P. Trouilloud, Y. Y. Wang, V. P. Dravid, and J. Z. Sun, Phys. Rev. B 54, R15629 (1996).

${ }^{13}$ N. D. Mathur, G. Burnell, S. P. Isaac, T. J. Jackson, B.-S. Teo, J. L. MacManus-Driscoll, L. F. Cohen, J. E. Evetts, and M. G. Blamire, Nature (London) 387, 226 (1997).

${ }^{14}$ N. D. Mathur, P. B. Littlewood, N. K. Todd, S. P. Isaac, B.-S. Teo, D.-J. Kang, E. J. Tarte, Z. H. Barber, J. E. Evetts, and M. G. Blamire, J. Appl. Phys. (to be published).

${ }^{15}$ R. A. Rao, D. Lavric, T. K. Nath, C. B. Eom, L. Wu, and F. Tsui, Appl. Phys. Lett. 73, 3294 (1998).

${ }^{16}$ A. J. Millis, A. Goyal, M. Rajeswari, K. Ghosh, R. Shreekala, R. L. Greene, R. Ramesh, and T. Venkatesan (unpublished).

${ }^{17}$ See, for example, A. Hubert and R. Schaefer, Magnetic Domains (Springer, New York, 1998).

${ }^{18}$ C. Kittel, Phys. Rev. 70, 965 (1946).

${ }^{19}$ A. Berger and H. Hopster, J. Appl. Phys. 79, 5619 (1996), and references therein.

${ }^{20}$ C. Kooy and U. Enz, Philips Res. Rep. 15, 7 (1960).

${ }^{21}$ In magnetic crystals, the spin-orbit interaction, which is at the microscopic origin of this resistivity anisotropy or anisotropic MR (AMR) effect, leads to a resistivity which depends not only on the orientation of magnetization and current and but also on the relation of these vectors to the crystal structure (see Ref. 22). For example, resistivity anisotropy of this type has recently been reported for transition metal epitaxial thin films (see Ref. 23).

${ }^{22}$ R. McGuire and R. I. Potter, IEEE Trans. Magn. MAG-11, 1018 (1975).

${ }^{23}$ U. Ruediger, J. Yu, L. Thomas, S. S. P. Parkin, and A. D. Kent, Phys. Rev. B 59, 11914 (1999).

${ }^{24} \mathrm{See}$, for example, Cullity, Introduction to Magnetic Materials (AddisonWesley, Reading, MA, 1972), p. 135.

${ }^{25}$ P. G. de Gennes, Phys. Rev. B 118, 141 (1960), and references therein.

${ }^{26}$ L. Brey (unpublished).

${ }^{27}$ L. Berger, J. Appl. Phys. 49, 2156 (1978). 